\title{
Modalités de travail en dyades et conduites à des tâches d'algèbre chez des élèves portugais
}

\section{Margarida Cesar, Anne-Nelly Perret-Clermont et Ana Benavente}

La recherche présentée ici se déroule dans le contexte de l'école obligatoire portugaise et des difficultés que connaît l'enseignement des mathématiques systématiquement confronté à une proportion importante d'élèves peu motivés pour cette discipline et en échec. Des interventions pédagogiques, construites comme des études de cas inspirées par un plan quasi-expérimental permettant des comparaisons, ont permis d'observer les réactions différentes d'élèves travaillant, seuls ou en dyades, à des problèmes d'algèbre. Il en ressort d'une part une meilleure compréhension du rôle de la modalité d'organisation des relations dyadiques dans la capacité à comprendre la tâche et à la gérer; et d'autre part une mise en évidence de l'engouement qui naît chez les élèves, embarqués dans des dialogues et des conflits socio-cognitifs, et qui alors modifient leurs attitudes à l'égard de ces mathématiques.

\section{Introduction}

Depuis une vingtaine d'années au Portugal, l'échec scolaire et les attitudes de rejet des élèves à l'égard des mathématiques, ont poussé les enseignants à chercher des solutions. En effet, les mathématiques, discipline présente dans toute la scolarité obligatoire (9 années), ont un poids considérable sur les choix professionnels ultérieurs. Mais il s'agit aussi de l'une des disciplines dont les élèves ont la représentation la plus négative ce qui les empêche même parfois d'essayer de collaborer, en classe, aux tâches que leurs professeurs leur proposent. Désimplication et démotivation caractérisent alors le paysage scolaire de cet enseignement.

La dernière réforme des curriculums a essayé de prendre en charge cette question en précisant non seulement les objectifs du point de vue des contenus mais aussi en termes de développement des compétences, des attitudes et des valeurs (Abrantes, Serrazina et Oliveira, 1999). En conséquence, les professeurs se trouvent confrontés à de nouvelles exigences, ce qui entraîne la nécessité pour eux de changer leurs pratiques pédagogiques mais sans qu'ils ne se sentent toujours pour autant préparés à répondre aux défis qui leurs sont ainsi posés. Vouloir changer 
ne signifie pas toujours savoir le faire. Comme toujours dans le domaine de l'éducation, les transformations sont lentes, les résistances nombreuses, et l'implication des professeurs est un facteur essentiel pour qu'une réforme du curriculum puisse réussir dans les faits. Toute mesure de démocratisation de l'école implique toujours la prise en compte du rôle des enseignants, producteurs et intervenants dans leurs espaces professionnel et social (Benavente, 1990).

Le contexte dans lequel le présent travail de recherche a commencé est celui précédant immédiatement cette dernière réforme du curriculum. L'ambiance y est favorable: des recherches existent déjà sur le travail en dyades ou en petits groupes; les professeurs se posent beaucoup de questions, et ils sont prêts à les adresser à d'autres spécialistes dans un contexte de collaboration interdisciplinaire. Mais la plupart des professeurs n'ont pas vécu eux-mêmes, en tant qu'élèves, le type d'expérience pédagogique qu'ils préconisent et ils ne bénéficient pas d'une formation professionnelle initiale qui les aurait préparés à la conduite de pratiques didactiques innovatives. En particulier ils ne sont pas habitués à l'idée que les élèves puissent tirer bénéfice d'interactions horizontales entre eux. En effet, au Portugal, jusque dans les années 70, l'enseignement des mathématiques a reposé sur un socle d'idées traditionnelles valorisant surtout l'acquisition d'automatismes par la répétition et la mémorisation, et ceci dans un contexte privilégiant les interactions verticales entre le professeur, maître de la relation didactique, et les élèves dont le rôle est perçu essentiellement comme réactif. Envisager une mutation des méthodes pédagogiques afin de faire plus de place à l'activité même de l'élève fait surgir alors trois types de questions: Comment implémenter un travail de groupe efficace, et comment faut-il s'y prendre pour que les différents élèves qui participent à une activité de groupe en tirent tous parti? Comment susciter des attitudes plus positives ou plus actives de la part des élèves dans les leçons de mathématiques? Comment les contenus mathématiques des leçons doivent-ils être articulés au développement des compétences des élèves?

Dans notre essai d'approfondir ces questions, la littérature sur le rôle des interactions sociales dans l'apprentissage et sur les démarches de travail de groupe en classe ont particulièrement retenu notre attention. Mais ce qui manque pour pouvoir élaborer des réponses pédagogiques concrètes avec ces enseignants, c'est une analyse de la variété des processus en jeu dans le contexte spécifique de leurs classes d'écoles portugaises avec des exemples à leur mettre sous les yeux. Notre intention a donc été alors essentiellement de comparer les effets de différents types de dyades, de tâches, de consignes, sur les modalités de travail et d'apprentissage d'élèves portugais dans leur milieu naturel. Dans cette première phase de recherche, l'approche choisie est donc celle d'observations systématiques organisées selon un plan quasi-expérimental. Elle sera suivie ultérieurement d'une deuxième phase consistant en une recherche-action avec les enseignants. L'approche s'inscrit donc dans une sorte de va-et-vient (Perret-Clermont 1980, 1982) entre la réflexion théorique que permet l'approche quasi-expérimentale, et 
la recherche de solutions pratiques, sur le terrain, en collaboration avec les professionnels concernés.

Comme l'a déjà constaté Benavente (1992), "les changements éducatifs sont lents». Changer ce que l'on enseigne et les manières de le faire, requiert des démarches difficiles, compliquées, avec beaucoup d'imprévus, impliquant des institutions, des groupes et des personnes, des relations, des pratiques, des normes, des pouvoirs, des intérêts, des savoirs, des lieux et des durées. Et c'est précisément parce que les changements en éducation sont lents qu'on ne peut pas perdre de temps». C'est pour commencer à les analyser que la première phase de ce travail, en situation de semi-laboratoire, grâce à l'appui des enseignants, examinera les effets d'un certain nombre de pratiques innovantes sur les capacités d'élèves portugais. Puis, toujours avec les enseignants, il s'agira ensuite d'examiner l'effet de l'implémentation de telles pratiques non plus en condition de semi-laboratoire mais dans la vie quotidienne des classes.

\section{Cadre théorique de référence}

Les recherches de différents auteurs (voir notamment à ce sujet Doise, Mugny et Perret-Clermont, 1975, 1976; Perret-Clermont 1979/96; Mugny, 1985) avaient déjà mis en évidence le rôle facilitateur d'interactions entre pairs pour le développement de compétences cognitives. Mais les premiers travaux, réalisés en s'appuyant essentiellement sur des épreuves psychologiques, souvent piagétiennes, avaient été effectués en situation de laboratoire. Or il s'est avéré rapidement que la transposition de leurs résultats dans des situations scolaires naturelles, et à propos de tâches issues du curriculum, posait des problèmes nouveaux (Brun, 1975; Schubauer-Leoni et Perret-Clermont, 1980, 1985, 1997) relatifs, notamment, à l'interprétation des consignes, au sens de la tâche, au "contrat didactique» implicite sous-jacent.

D'autre part, dans l'étude de Schubauer-Leoni et Perret-Clermont (1980) de même que dans les recherches de César (1994a, 1994b, 1995) différentes formes d'interactions et de communication avaient retenu l'attention. Si l'efficacité comparativement plus grande du travail en dyades sur le travail individuel tendait souvent (mais pas toujours) à se confirmer, apparaissait aussi le rôle du facilitateur que pouvait avoir, du moins dans certaines circonstances, le recours à une troisième personne (adulte ou pair) placée dans un rôle de «décodeur», c'est-àdire devant prendre connaissance du travail réalisé et en dire le sens. Tout semble se passer comme si les interactions entre pairs étaient susceptibles de faciliter le travail de réflexion mais ceci d'autant plus si son fruit doit être communiqué à un troisième partenaire devant le comprendre. Ces recherches montraient aussi l'absence d'une relation directe entre le niveau opératoire des sujets et leur réussite dans différentes tâches (Brun, 1975; César et Esgalhado, 1986, 1987, 1988, 1991; César, Camacho et Marcelino, 1991). Dans les dernières études citées, un 
niveau opératoire élevé apparaissait comme une condition nécessaire mais non suffisante pour obtenir une bonne réussite scolaire.

Des recherches des années ' 80 mettent donc en évidence le caractère facilitateur du travail en dyades pour les progrès cognitifs, en particulier dans des tâches mathématiques (Ames et Murray, 1982; Balacheff, 1982; Botelho, 1991; Flieller, 1986, 1990; Carugati et Mugny, 1985; Gilly et Roux, 1984; Gilly, Fraisse et Roux, 1988; Grossen et Perret-Clermont, 1984; Perret-Clermont et Nicolet, 1988; Schubauer-Leoni, 1990; Schubauer-Leoni et Perret-Clermont, 1980, 1985). Pourtant il existe également des données contraires dans lesquelles on n'observe pas l'effet bénéfique du travail en dyades (ainsi par exemple dans le cas des travaux de Tudge, 1985, 1989). Et surtout, la diversité des consignes données aux dyades, des types de tâches choisies, des méthodologies d'observation utilisées, etc., rendent difficile un bilan général qui affirmerait péremptoirement que le travail en dyades est toujours supérieur au travail individuel. Il s'agit de mieux comprendre les processus en jeu. Ainsi, certaines recherches comparent l'efficacité relative des dyades symétriques et asymétriques (Balacheff et Laborde, 1985; Blaye 1987, 1988), des dyades interagissant pleinement et des dyades ne faisant que travailler individuellement côte à côte, ou encore des dyades comprenant (comme dans la situation scolaire classique) deux élèves silencieusement assis l'un à côté de l'autre, seulement autorisés à regarder éventuellement le travail d'autrui mais sans en parler (Blaye, 1987). Ont été examinés également différentes modalités de prises de rôle de la part du décodeur (Balacheff, 1982; Schubauer-Leoni et Perret-Clermont, 1985); ou l'effet d'associer à la coopération intra-dyades une compétition inter-dyades (Johnson et Johnson, 1989; Slavin, 1980, 1990; Nicolet 1995). Globalement la prise de connaissance de cette littérature étaye l'hypothèse selon laquelle, en général, les résultats sont meilleurs lorsqu'il s'agit de dyades pleinement en interaction, qui bénéficient de pairs décodeurs, et de situations dans lesquelles un enjeu compétitif est associé à la nécessité interne de coopérer. Par contre les résultats sont contradictoires en ce qui concerne les bénéfices comparés des dyades symétriques et asymétriques.

C'est aussi dans les années '80 qu'a grandi la prise de conscience du rôle du contexte et de l'intérêt d'étudier les conduites cognitives comme socialement situées. Parallèlement on redécouvre Vygotsky et l'éclairage qu'il apporte quant au rôle structurant du social et des médiations symboliques dans le développement cognitif. Différents auteurs réalisent alors des études qui, notamment, comparent les compétences mathématiques des enfants à l'école et dans la vie quotidienne (Carraher, Carraher et Schliemann, 1989; Säljö et Wydham, 1987; Saxe, 1989). Ils mettent en évidence les stratégies de résolution de problèmes auxquelles les sujets recourent dans ces différents cas et qui permettent de comprendre pourquoi certaines réussites, dans des situations de vie, s'accompagnent néanmoins d'échecs à l'école dans des tâches qui, aux yeux des pédagogues, avaient pu paraitre semblables. Ces travaux mettent en relief l'activité interprétative du sujet qui ne résout une tâche qu'en lui attribuant un sens, lequel va dé- 
pendre des circonstances. Deux tâches mathématiques, apparemment semblables, peuvent présenter des degrés de difficulté variables en fonction du sens que les élèves parviennent ou non à leur attribuer. «Toute réponse (...) est indissociable des conditions sociales dans lesquelles elle est donnée, c'est-à-dire de ses conditions de production»(Grossen, 1988, p. 365).

Une autre série d'études attire l'importance sur le statut de celui qui sollicite la compétence de l'enfant, ainsi que de la mise en scène dans laquelle il se présente (Donaldson, 1978; Light, 1986; Light et al. 1987; Schubauer-Leoni, 1990; Grossen, 1988; Iannaconne, 1990; Säljö et Windhamn, 1987; Säljö, 1991). Le niveau de performance de l'enfant et les stratégies qu'il met en œuvre sont susceptibles de différer selon que son partenaire se présente comme un enseignant, un expérimentateur, un conteur, etc. L'importance de la situation de questionnement est également mise en évidence dans le cas particulier où il s'agit de traiter de problèmes absurdes (Martins et Neto, 1990; Giosué, 1991). Dans le prolongement de ces observations apparaît alors l'importance du "contrat didactique» (Brousseau, 1988; Chevalard et al., 1988; Schubauer-Leoni, 1986a, 1986b; Schubauer-Leoni et Perret-Clermont 1997). On observe effectivement que la relation maître-élève est gérée par une série de règles implicites (Forman, 1989) qui légitiment les attentes des uns et des autres, définissent les rôles respectifs, permettent de construire l'objet de discours commun et de gérer une attention conjointe sur les mêmes actions et opérations.

Ainsi, au fur et à mesure que les recherches sur les processus de compréhension de l'enseignement des mathématiques, et plus largement sur le rôle des interactions sociales et des communications dans l'appropriation de connaissances, s'affinent, on voit muter le paradigme de référence: une première génération de recherches pose ces questions en termes de facteurs indépendants externes (nature de la tâche, consignes de travail, caractéristiques des dyades, etc.) susceptibles d'affecter le niveau des performances cognitives ou scolaires des sujets; tandis que la deuxième génération d'études reformule cette problématique en mettant l'accent sur les caractéristiques des processus de communication, et sur l'insertion sociale des interactions qui permettent (ou non) aux partenaires de construire un objet commun de discours et de réflexion, voire d'apprentissage (Perret-Clermont, Perret et Bell 1991; Pléty, 1996; Grossen et Py, 1997; Kumpulainem et Mutanen, 1999; Peixoto et Monteiro, 1999; César, 2000c; van der Linden et al., 2000). Cette dernière perspective rejoint d'ailleurs les propos de Piaget, en 1965 déjà, sur la nécessité qu'ont les sujets, pour se comprendre, de partager des signes ou des significations communs; ou ceux de Rommetveit (1976) ou de Wertsch (1991) sur la construction de l'intersubjectivité entre les locuteurs, indispensable pour qu'ils puissent se comprendre et, à partir de là, construire éventuellement de nouvelles connaissances.

Smith (1996, p. 118) met en évidence encore un autre aspect qui nous semble essentiel: «une communication réussie ne nécessite pas que les deux partenaires qui prennent part à l'échange acceptent le point de vue de l'autre puisqu'il est 
possible qu'il s'agisse d'un désaccord rationel. Nous dirons plutôt qu'une communication est réussie si chacun comprend la pensée de l'autre». ${ }^{1}$ Il s'agit alors de conflits socio-cognitifs: être, dans l'ici et le maintenant, confrontés à la pensée d'autrui oblige à réfléchir la perspective propre en relation avec des alternatives et, par la même, à en approfondir la compréhension voire à la remettre en cause.

Enfin la relecture de la théorie deVygotsky invite aussi à une prise en compte de la zone proximale de développement (par ex. Rogoff et Wertsch, 1984; Moll, 1990; Allal et Ducrey, 2000): un élève bénéficie de l'interaction avec un pair plus compétent seulement lorsque celui-ci le rejoint dans sa zone de développement potentiel. Pourtant, dans d'autres études citées ci-dessous, la simple interaction avec un pair, même moins compétent, est susceptible d'être bénéfique sur le plan cognitif. Alors quel est le rôle de ce partenaire?

\section{Investigations}

\section{Démarche et population}

L'objectif de la démarche de l'étude présentée ici, et qui s'est déroulée sur deux ans, est d'examiner, à travers des études de cas, dans des classes d'école portugaises, deux hypothèses psycho-pédagogiques générales, à savoir que le travail en dyades est susceptible de susciter, en général, de meilleures performances que le travail individuel (Hypothèse 1), et de faire progresser, sur le plan individuel, les élèves entre le pré-test et le post-test (Hypothèse 2). Il s'agit aussi d'examiner si travail individuel ou en dyades suscite le recours à des stratégies de résolution différentes; et si la modalité de relation imposée aux dyades affecte les progrès des sujets sur des tâches mathématiques; de voir enfin si les consignes de travail données aux élèves affectent leurs interactions et leurs productions.

Tous les sujets sont soumis aux mêmes tâches, inspirées par le programme d'enseignement des mathématiques - en fait plus spécifiquement des équations - en 7e année. Ils appartiennent à des classes portugaises de l'enseignement régulier diurne. Chaque étude de cas consiste en l'observation de classes différentes soumises à des conditions de travail (dites "conditions expérimentales» différentes) et dont les caractéristiques sont résumées plus loin dans le tableau 1. Pour consolider les résultats, la récolte de données de la seconde année d'études a été organisée en répliquant les deux conditions qui se sont avérées les plus fructueuses (les dyades avec interaction; et les dyades simultanément en compétition inter-dyades et coopération intra-dyades).

La recherche s'est déroulée dans dix classes (227 élèves) de 7e année scolaire d'une école officielle de la zone de Lisbonne. Cette école a été choisie parce qu'elle recrute une population hétérogène du point de vue socio-économique et culturel, et parce qu'elle connaît un taux d'échecs scolaires et de difficultés en mathématiques conforme à la moyenne de la population scolaire portugaise. $\mathrm{Ne}$ sont pas inclues dans l'étude les classes relevant de l'enseignement spécial, ni 
celles comprenant beaucoup d'élèves dont l'âge s'avère nettement supérieur à l'âge officiel de la 7ème année. Les classes ont en moyenne 28 à 30 élèves. Pour l'analyse des données on a dû laisser cependant tomber les élèves qui n'ont pas passé toutes les épreuves.

\section{Instruments}

Deux types de tâches sont utilisées, toutes en lien avec le chapitre du programme relatif aux équations. Un premier type de tâches, dites «habituelles» $(\mathrm{H})$, ont été jugées par les professeurs comme typiques de celles qu'ils utilisent en classe. Dans la littérature, ces tâches sont souvent désignées comme "des exercices» puisque les élèves doivent y procéder à l'application des règles de résolution des équations qu'ils ont apprises antérieurement lors des leçons de mathématiques. Une étude pilote a permis de tester ces exercices et d'en faire deux versions de difficultés semblables ( $\mathrm{H} 1$ et $\mathrm{H} 2)$ : la première est soumise aux élèves en pré-test et la deuxième en post-test.

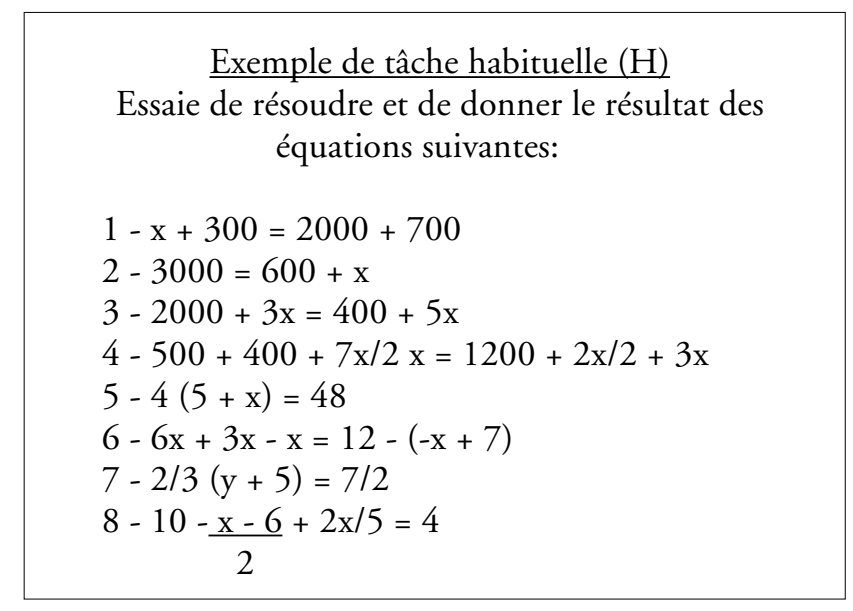

L'autre type de tâches, «non-habituelles», (NH) correspond à ce que la littérature désigne comme des "problèmes». Il s'agit de tâches à résoudre en se référant à la métaphore de la balance, métaphore qui, au moment de la présente étude (réalisée avant la mise en application des nouveaux curriculums scolaires), n'est pas encore utilisée dans les manuels portugais disponibles ni par les enseignants. Ces problèmes sont présentés selon un ordre de difficulté croissant. Tant les tâches «habituelles» que «non-habituelles» ont donc porté sur des équations du premier degré, typiques du curriculum de 7e année. 


\section{Exemple de tâche non-habituelle (NH)}

Essaie de répondre aux questions suivantes en expliquant toujours comment tu as fait. Merci pour ta collaboration.

1 - Monsieur Costa a une épicerie dans un petit village qui s'appelle S. António de Penacova. Sa fille est en 7e année scolaire et elle est en train d'apprendre a résoudre des problèmes. Monsieur Costa n'est jamais allé à l'école secondaire mais il a décidé qu'il allait aider sa fille. Alors, il a pris une balance à deux plateaux qu'il avait encore à l'épicerie et une boîte avec des poids. Les poids variaient entre 250 grammes et 10 kilos. Dans chaque cas réponds à la question qu'on te pose et explique toujours comment tu es arrivé à ce résultat. Tu peux utiliser n'importe quel type de représentation.

a) - Sur le plateau gauche je mets un poids de plus d'un demi-kilo; sur le plateau droit je mets trois kilos. La balance est équilibrée. Combien pèse le poids que j'ai mis à gauche?

b) - Sur le plateau gauche je mets 5 poids tous égaux et pour équilibrer la balance je dois mettre sur l'autre plateau un poids de trois kilos avec un autre poids de 750 grammes. Combien pèse chacun des poids que j'ai mis sur le plateau gauche?

c) - Je choisis 8 poids égaux, et je mets 6 poids sur le plateau gauche et 2 sur le plateau droit. Pour équilibrer la balance je dois encore mettre deux kilos de plus sur le plateau droit. Combien vallait chacun des poids que j'avais initialement choisis?

d) - Sur le plateau gauche j'ai un poids de 6 kilos et sur le plateau droit je vais mettre une série de poids de même taille. Mais quand j'arrive à $22 \mathrm{de}$ ces poids, je remarque que je n'ai plus assez de ces poids et que la balance n'est pas encore équilibrée. Je sais que si je pouvais retirer aux 6 kilos deux poids égaux à ceux du plateau droit, j'aurais la balance en équilibre. Combien vallait chacun de ces poids du plateau droit?

C'est un professeur de mathématiques inconnu des élèves qui fait passer les sessions initiale et finale: pré-test et post-test. Les sujets répondent individuellement et leurs performances sont classées sur une échelle en trois niveaux: faible, moyen et élevé. Les 1 à 3 sessions intermédiaires au cours desquelles les élèves sont invités à résoudre les tâches «non habituelles» sont dirigées par une expéri- 
mentatrice (une psychologue extérieure à l'école), selon des consignes qui varient en fonction des conditions expérimentales de chaque étude (voir le tableau 1). La session intermédiaire (NH2) est enregistrée. Lors de chaque session, on récolte les feuilles de réponse des élèves qui ont été priés d'écrire tout ce qu'ils pensent utile à la réponse et de ne pas effacer mais de simplement tracer les éléments de leurs réponses qu'ils pourraient vouloir modifier.

\section{Conditions de travail ("conditions expérimentales») de chaque étude de cas}

Le tableau 1 décrit les différentes conditions dans lesquelles les enfants ont été invités à travailler dans chaque étude de cas.

Ces différentes études de cas ont été conçues dans le but de conduire une enquête pilote d'observation qualitative de l'influence de la modalité de constitution des dyades, et des consignes qui leur étaient données, sur leurs interactions, leurs performances ainsi que sur les progrès subséquents de leurs membres. Au départ de cette enquête dans les classes portugaises, les chercheurs n'avaient pas d'hypothèses suffisamment précises (ni de budget!) pour envisager à ce stade d'interroger de grands nombres de sujets dans chaque cas. Nous présentons maintenant les conditions expérimentales de chacune de ces études:

Première année d'enquête:

- Etude de cas $N^{\circ} 1$ :

Deux conditions: dyades symétriques versus asymétriques quant aux niveaux respectifs des sujets au pré-test

- Etude $N^{\circ} 2$ :

Trois conditions de dyades travaillant selon différentes modalités de collaboration:

1) Les enfants sont invités à répondre ensemble sur la même feuille en interagissant, sans que l'expérimentatrice n'impose de contraintes à leur modalité de relation et de communication (DI).

2) Les enfants commencent par répondre séparément et ensuite doivent se mettre d'accord sur la meilleure des deux réponses qui sera alors fournie à l'expérimentatrice.

3) Les enfants commencent par répondre séparément, c'est-à-dire individuellement, et ensuite élaborent une réponse collective sur une troisième feuille qu'ils remettent à l'expérimentatrice.

- Etude $N^{\circ} 3$ :

Quatre conditions:

1) Les dyades travaillent ensemble (DI).

2) Les élèves d'une dyade travaillent côte à côte sans interaction verbale et en pouvant seulement regarder ce que fait l'autre mais sans en parler (DSI).

3) Chaque élève travaille individuellement.

4) Un groupe contrôle est constitué d'élèves ne faisant pas ces tâches. 


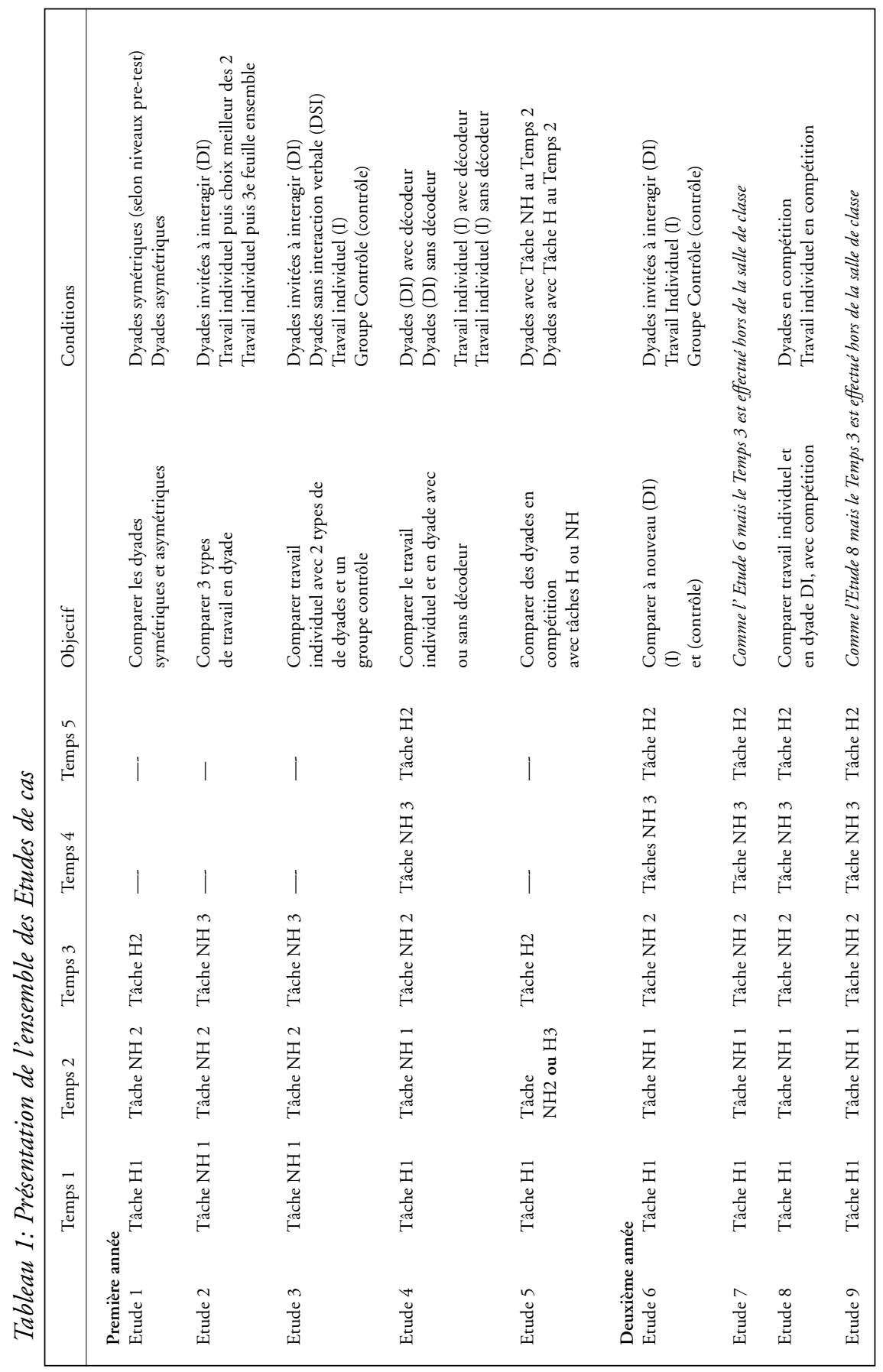




\section{- Etude $N^{\circ} 4$ :}

Dyades (DI) ou individus, devant ou non, faire lire le résultat de leur travail par un pair décodeur (c'est-à-dire dont le rôle est d'expliciter quelles sont la tâche et la solution trouvée).

\section{- Etude $N^{\circ} 5$ :}

Les sujets des dyades (DI) sont invités à travailler ensemble dans une situation de compétition avec une autre dyade. Ensuite les deux dyades confrontent leurs résultats et décident quelle est la meilleure performance. La moitié des dyades travaillent aux tâches NH2, et l'autre moitié aux tâches H3.

Deuxième année d'enquête:

- Etude $N^{\circ} 6$ :

Reprise partielle et extension de l'Etude $N^{\circ} 3$ :

1) Les dyades travaillent ensemble (DI).

2) Chaque élève travaille individuellement.

3) Un groupe contrôle est constitué d'élèves ne faisant pas ces tâches.

\section{- Etude $N^{\circ} 7$ :}

Reprise de l'Etude $N^{\circ} 6$ mais le temps 3 est effectué hors de la salle de classe.

\section{- Etude $N^{\circ} 8$ :}

Reprise partielle et extension de l'Etude $N^{\circ} 5$ :

1) Les sujets des dyades (DI) sont invités à travailler ensemble dans une situation de compétition avec une autre dyade. Ensuite les deux dyades confrontent leurs résultats et décident quelle est la meilleure performance.

2) Les individus travaillent seuls dans une situation où les sujets se croient en compétition avec un autre individu.

\section{- Etude $N^{\circ} 9$ :}

Reprise de l'Etude $N^{\circ} 8$ mais le temps 3 est effectué hors de la salle de classe.

\section{Résultats}

L'observation de différentes stratégies de résolution On observe, chez les sujets, différentes stratégies de réponse qui semblent largement correspondre au degré de difficulté des problèmes à résoudre, pour lesquels elles se révèlent plus ou moins adaptées. L'analyse permet d'identifier, pour les tâches «non-habituelles», 5 stratégies de réponse:

1. La stratégie de représentation graphique à l'aide de dessins et de schémas pour représenter les données du problème.

2. La stratégie de «manipulation de variables» (dénommée ainsi par Carraher, 
Carraher et Schliemann, 1989): stratégie de simplification qui vise à rendre le problème plus simple, par exemple en supprimant les poids équivalents dans les plateaux de droite et de gauche.

3. La stratégie de résolution par essais et erreurs, en procédant par hasard ou de façon systématique.

4. La stratégie de résolution arithmétique avec le recours à l'utilisation des 4 opérations de base.

5. La stratégie de résolution algébrique à l'aide d'équations.

Il convient de noter que les deux premières stratégies sont des stratégies de réponse, mais non de résolution, aussi n'aboutissent-t-elles à une solution du problème que si elles sont associées à l'une des 3 autres stratégies mentionnées. La représentation graphique et la manipulation de variables ne sont en fait que des stratégies intermédiaires, qui facilitent la découverte d'une stratégie de résolution.

La stratégie de représentation graphique s'avère utilisée par la plupart des sujets dans la première et troisième tâche non-habituelles, qui sont celles qui n'ont pas les balances dessinées.

Il est intéressant d'observer (César, 1994a) que la majorité des sujets utilisent les stratégies de résolution arithmétique lorsqu'ils cherchent à résoudre les problèmes les plus simples. Néanmoins, certains recourent à des stratégies de type additif, d'autres utilisent celles de type soustractif. On constate que ce sont ces derniers qui sont les plus susceptibles de progresser du point de vue des stratégies de résolution au cours de l'expérience. Le mode de traitement des problèmes les plus simples semble être un bon prédicteur de la gestion ultérieure des problèmes plus complexes.

Les stratégies de résolution par essais et erreurs permettent de gérer les problèmes d'une complexité moyenne mais ne conviennent pas pour la résolution des problèmes ultérieurs plus complexes.

On constate aussi que les stratégies algébriques sont rarement utilisées dans les problèmes les plus simples, si ce n'est par quelques sujets connus pour leurs bons résultats scolaires. Le recours à ces stratégies algébriques semble réservé surtout aux problèmes les plus complexes nécessitant le maniement de deux types différents de variables.

La manipulation de variables est une stratégie rarement observée, seulement dans les problèmes plus complexes. Elle aboutit presque toujours à une résolution correcte. Elle est souvent associée aux stratégies de résolution arithmétique. Elle s'avère surtout choisie par les sujets qui travaillent en dyades, en particulier lors de la tâche NH3.

La confrontation aux hypothèses initiales

Cette expérience était donc sous-tendue par deux hypothèses psycho-pédagogiques générales, à savoir d'une part que les sujets qui travaillent à deux sont sus- 
ceptibles d'avoir de meilleurs résultats que ceux qui travaillent individuellement (hypothèse 1); et d'autre part que les sujets qui travaillent à deux sont susceptibles de faire preuve de plus de progrès lors des post-tests (hypothèse 2).

Tableau 2: Comparaison des niveaux des performances collectives et des performances individuelles aux tâches "non habituelles» (NH2) des Etudes de cas $N^{\circ} 1$ à 9

\begin{tabular}{lllll}
\hline & \multicolumn{3}{l}{ Niveau de la performance } & \multirow{2}{*}{ N } \\
\cline { 2 - 4 } & Faible & Moyen & Elevé & \\
\hline Condition 1 (D): Perfo. dyades & 16 & 23 & 41 & 160 (80 dyades) \\
Condition 2 (I): Perfo. indiv. & 17 & 19 & 15 & 51 \\
\hline
\end{tabular}

Légende: Perfo. dyade $=$ performances collectives des dyades

Perfo. indiv. $=$ performances des sujets travaillant individuellement

Hypothèse1: Perfo. dyades > Perfo. individuelles, $\mathrm{p}=.03$

Test avec la procédure de Jonckheere (Jonckheere, 1954; Leach, 1979; Pochon, 1991 et 1997) à partir du $S$ de Kendall.

Tableau 3: Comparaison de l'évolution des performances des sujets sur les "tâches habituelles", entre le pré-test (H1) et le pos-test (H2), selon la condition expérimentale du travail lors de NH2 des Etudes de cas 1,4,5,6,7,8 et 9.

\begin{tabular}{lccr}
\hline & \multicolumn{2}{l}{ Evolution des performances } & N \\
\cline { 2 - 3 } & Non progrès & Progrès & \\
\hline Condition 1 (D): & 75 & 25 & 100 \\
Condition 2 (I) & 29 & 6 & 35 \\
Condition 3 (contrôle) & 15 & 1 & 16 \\
\hline
\end{tabular}

\footnotetext{
Légende: $\mathrm{D}=$ sujets ayant travaillé en dyades

$\mathrm{I}=$ sujets ayant travaillé individuellement contrôle: sujets n'ayant pas fait $\mathrm{NH} 2$

Hypothèse 2: $\mathrm{C} 1>\mathrm{C} 2>\mathrm{C} 3, \mathrm{p}=0.05$

Test avec la procédure exacte de Jonckheere (Jonckheere, 1954; Leach, 1979; Pochon, 1991 et 1997)

à partir du $\mathrm{S}$ de Kendall.
}

On observe effectivement que les résultats confortent ces deux hypothèses: si l'on prend en compte tous les sujets de toutes les études de cas 1 à 9 (donc toutes "conditions expérimentales» confondues), on peut voir (tableau 2) que les performances des dyades sont meilleures que celles des individus; quant aux données du tableau 3, qui prennent en compte les sujets des études de cas 1, 4, 5, 6, 7,8 et 9, c'est-à-dire celles comprenant pré- et post-tests, elles révèlent des progrès supérieurs chez les sujets ayant exercé les tâches NH, en particulier dans les dyades. Donc, dans ces conditions scolaires portugaises, le travail en dyades s'avère globalement plus efficace que le travail individuel tant pour la performance collective autour des tâches «non habituelles» que pour les progrès ultérieurs lors des post-tests autour des tâches «habituelles». 


\section{Approche descriptive de l'influence des différents types de dyades et de consignes}

Les élèves travaillaient donc, dans chaque Etude de cas, dans des conditions expérimentales qui se différenciaient les unes des autres selon les modalités décrites plus haut et rappelées dans le tableau 1. Qu'ont-elles permis d'observer ? (Rappelons que ces observations ici ne visent pas un résultat statistique vu les petits effectifs de sujets).

Dans l'Etude $N^{\circ} 1$, il apparaît que les élèves travaillant avec un camarade ayant présenté une performance individuelle de même niveau au pré-test (dyades symétriques) et ceux ayant collaboré avec un pair de niveau différent (dyades asymétriques) ne se différencient pas quant à leurs performances collectives ou leurs progrès individuels ultérieurs. Mais l'examen attentif des enregistrements du travail en dyades fait apparaître des différences qualitatives quant aux modalités de celles-ci: dans les dyades asymétriques les sujets interagissent plus et de façon plus variée: conflits socio-cognitifs, propos sur la gestion des relations (qui doit agir, quand, comment parvenir à un consensus), argumentations diverses. Par contre il vaudrait la peine de vérifier dans une étude ultérieure si les sujets qui bénéficient le plus des interactions en dyades symétriques ne sont pas ceux qui avaient présenté au pré-test les niveaux inférieurs.

Dans l'Etude $N^{\circ} 2$, il semble que seuls les sujets de niveau moyen interagissant en condition DI (c'est-à-dire sans contrainte imposée par l'expérimentatrice quant au mode de relation et communication) font des progrès au post-test. Par contre on ne trouve pas de différences au niveau des performances collectives. L'observation des interactions montre qu' effectivement la consigne influence leur déroulement: lorsque les sujets doivent simplement choisir la meilleure des deux feuilles, ils n'interagissent presque pas semblant se borner à chosir la réponse de l'élève ayant la meilleure réputation scolaire! Et cela ne les fait guère progresser bien sûr. Au contraire on voit même un élève régresser après n'avoir guère investi son travail puisqu'il savait qu'ensuite la performance du bon élève s'imposerait. Par contre on retrouve à nouveau le cas d'une dyade symétrique d'élèves ayant eu des niveaux très faibles au pré-test et qui progressent ensuite: lors de l'interaction ils ont dû longuement discuter pour parvenir à choisir la réponse ce qui leur a valu d'envisager aussi des réponses alternatives. La consigne exigeant des élèves qu'ils produisent ensuite, ensemble, une troisième réponse n'a pas eu l'effet attendu, les élèves semblant préférer se borner à repérer laquelle des deux réponses existantes ils recopieraient sur la 3ème feuille, se retrouvant donc, de fait, dans la même condition expérimentale que l'autre groupe.

Les résultats de l'Etude $\mathrm{N}^{\circ} 3$ montrent l'intérêt d'une reprise ultérieure de ses conditions expérimentales avec un plus grand nombre de sujets: il semble en effet que seules les dyades interagissant en condition DI (sans contrainte) voient leurs membres progresser, tandis que les sujets travaillant simplement côte à côte ne se débrouillent pas mieux (voire moins bien) que les élèves travaillant individuellement (confirmant par là le savoir traditionnel des enseignants qui n'attendent rien de bon des élèves qui copient sur leur voisin). 
Dans l'Etude $N^{\circ}$ 4, le recours a un pair décodeur n'entraîne pas d'effets. D’une part les élèves ne semblent pas être affectés par une éventuelle anticipation de sa présence, et d'autre part ils s'engagent, quelque soit la condition expérimentale, d'emblée dans la tâche que leur performance y atteint sans doute un plafond.

Les résultats de l'Etude $\mathrm{N}^{\circ} 5$ sont les plus nets: c'est dans la condition où les sujets travaillent en dyades lors d'une compétition (même s'ils n'y reçoivent pas de prix et y sont leurs propres juges) avec une autre dyade qu'ils performent le mieux et cette expérience collective, même très brève, les fait progresser ensuite individuellement. Le fait d'avoir interagi autour d'une tâche habituelle (H3) ou inhabituelle (NH2) ne semble pas avoir affecté les performances. Mais les interactions ont été plus riches autour de la tâche NH.

Les Etudes $N^{\circ} 6$ et 7 ont confirmé que la condition de travail en dyade dans une situation d'intercation normale (DI) était plus favorable que le travail individuel, que ce soit dans ou hors de la classe. Les Etudes $\mathrm{N}^{\circ} 8$ et 9 ont confirmé l'effet stimulant de la compétition.

\section{Discussion générale}

Une première observation dans cette enquête nous encourage dans notre démarche: c'est l'enthousiasme manifesté par les élèves! Alors que nombre d'entre eux étaient démotivés en mathématiques, peu studieux dans cette branche dans laquelle ils tendaient à obtenir des résultats faibles, ils ont répondu très positivement à nos sollicitations, pourtant répétées, dans le cadre de cette recherche. Certes il est bien connu qu'un effet «expérimentation» est suceptible de se produire lors de toute recherche à l'école en raison de la nouveauté de la démarche, de la rupture qu'elle crée dans les routines quotidiennes, etc. Cependant nous avons néanmoins été surprises par l'engouement et la ténacité des élèves. Certains guettaient notre arrivée, suppliaient d'avoir leur tour (il a fallu organiser des séances supplémentaires hors plan expérimental pour les contenter), et la plupart restaient volontairement bien au-delà de l'horaire scolaire (y compris au prix, parfois, de sauter un repas). Il nous a semblé que la motivation ne tenait pas qu'à la nouveauté mais aussi au fait de bénéficier de l'attention d'une adulte sincèrement intéressée à leurs conduites réflexives et à leurs difficultés mais aussi aux compétences en mathématiques. Et un goût pour les mathématiques a même commencé à voir jour. Bien sûr cela n’a pas manqué d'intriguer les enseignants qui, à leur tour, se sont penchés sur l'expérience.

Nous avons également pu obsever l'effet mobilisateur des tâches non habituelles (NH), pourtant fort simples, que nous avions retenues. En effet Vergnaud (1986) avait attiré notre attention sur l'importance de soumettre aux élèves de réels problèmes lorsqu'on veut qu'ils se mobilisent vraiment dans l'appropriation de compétences mathématiques: «(...) aussi bien par ses aspects pratiques, que 
part ses aspects théoriques, le savoir se forme à partir de problèmes à résoudre, ce qui veut dire, de situations à dominer (...) Cela devrait être vrai également dans l'étude des mathématiques, mais ce n'est pas ce qui se vérifie. La tendance la plus courante est d'apprendre "des manières de faire" ou des algorithmes, reliant ces problèmes à des classes relativement limitées» (p. 76). Les tâches non habituelles que nous avons présentées aux élèves semblent avoir en effet appelé un fort engagement de leur part et suscité des interactions plus riches. Il est particulièrement intéressant de noter que, bien que le travail en dyades ait porté sur ces tâches non habituelles, il en soit résulté des progrès subséquents des sujets sur les tâches habituelles.

\section{Le recours à différentes stratégies de résolution}

Cette enquête met en évidence des stratégies de résolution de la part des élèves différentes selon la difficulté de la tâche et leurs conditions sociales de travail.

Certains élèves ne recourent qu'à des stratégies arithmétiques mais celles-ci ne s'avèrent adaptées que pour les problèmes les plus simples et s'ils s'accrochent à elles ils ne pourront pas résoudre les problèmes plus complexes. Notons que cette fixation s'observe surtout chez les sujets travaillant individuellement alors qu'en dyades, plus fréquemment, les stratégies s'adaptent au niveau de difficulté des problèmes. Ce sont les dyades qui font le plus recours aux stratégies de manipulation de variables, c'est-à-dire aux stratégies les plus efficaces pour des résolutions correctes. On observe aussi que lorsque des membres d'une dyade découvrent ensemble une stratégie, ils sont en général susceptibles de la remobiliser quand ils se retrouvent ultérieurement seuls.

A propos de cette stratégie de manipulation de variables, il est amusant de constater que bien qu'elle soit la plus adaptée aux problèmes complexes, elle rencontre une certaine résistance chez les élèves qui hésitent à l'utiliser car ils pensent que «ce n'est pas de la mathématique»! En effet l'écoute des enregistrements de leurs interactions orales révèle que parfois les enfants l'utilisent pour résoudre le problème mais évitent de la mettre par écrit sur la feuille qui sera rendue à l'expérimentatrice! Car ils pensent qu'elle n'est pas acceptée par l'école dans la cadre sérieux et formel des mathématiques! Ceci nous rappelle la recommandation vigoureuse de Carraher, Carraher et Schliemann (1989) qui ont, eux aussi, observé l'importance des stratégies de résolution «spontanées» des enfants (fussent-elles acquises par la culture enfantine ou celle des rues) et le gain qu'il peut y avoir à les explorer en détail et à en tirer parti dans l'enseignement plutôt qu'à les ignorer voire les réprimer et les faire passer dans la clandestinité.

\section{Efficacité et effet subséquent de l'interaction sociale} Les données récoltées sont venues confirmer nos deux grandes hypothèses psycho-pédagogiques de départ à savoir d'une part que le travail en dyades était suceptible de conduire à des performances collectives meilleures que celles des individus isolés, mais aussi que ces interactions étaient suceptibles d'induire des 
progrès dans les compétences individuelles ultérieures à traiter seuls ces problèmes ou d'autres exercices plus traditionnels. Cependant, comme prévu, et comme d'autres travaux l'ont encore montré parallèlement (par ex.: Forman et Larreamendy-Joerns, 1995; Howe et al., 1995) toutes les modalités de travail en dyades ne présentent pas cette fécondité: il ne suffit pas de placer les enfants deux par deux car le type d'instructions, la modalité d'interaction sucitée, la composition de la dyade notamment affectent les processus en jeu et dans certains cas (par exemple lorsqu'une réelle interaction n'est pas possible car les compagnons ne peuvent que s'observer mais pas se parler) les effets sont même néfastes.

Il semble que la chose importante soit la possibilité (voir l'invitation à) de discuter des stratégies de résolution jusqu'à l'élaboration d'un réel consensus, en évitant des incitations à un leadership impositeur ou à une soumission passive au prestige d'un partenaire réputé plus capable. Les situations les plus prometteuses sont celles où les partenaires doivent réaliser le plus grand effort de décentration pour parvenir à prendre en compte le point de vue d'autrui, expliciter leurs conjectures, gérer leurs conflits socio-cognitifs, et pour cela il faut que la tâche soit suffisamment difficile que pour être intéressante mais pas trop (Healy, Hoyles et Sutherland, 1990) Cela rejoint d'autres observations (Perret-Clermont, 1979/1996).

L'invocation d'une situation de compétition (inspirée par Slavin, 1980 et 1990; Nicolet, 1994) a mobilisé fortement les enfants: leur rythme de travail plus rapide et leur persévérance ont porté des fruits tant au niveau de la performance collective que des progrès individuels aboutissant aux meilleurs résultats mathématiques de notre échantillon.

\section{Considérations finales}

Cette recherche a montré à nouveau que, dans certaines conditions, le travail avec un pair, même pendant une durée très brève, est susceptible d'être fort profitable. L'enquête nous a permis de repérer certaines caractéristiques, importantes pour la réussite pédagogique, du type de dyade et de consigne et ouvre ainsi la voie à des actions en classe nouvelles dont on espère qu'elles pourront contribuer à élever les taux de réussite dans cette discipline scolaire importante.

$\mathrm{Au}$ Portugal, la disposition spatiale la plus fréquente en classe est fondée sur des tables à deux places. Cependant, dans la majorité des cas, l'enseignement ne favorise pas pour autant les interactions horizontales entre pairs: ce sont les interactions verticales qui prévalent, avec un fort contrôle par le professeur de tout le processus interactif. Nous sommes en train de faire l'expérience que la diffusion des résultats de la présente recherche, leur discussion avec les enseignants, l'essai de les implémenter en classe, sont susceptibles de stimuler l'action pédagogique en vue d'une amélioration des capacités des élèves et d'une modification de leurs attitudes à l'égard des mathématiques (César, 1997, 1998, 2000 a et b; César et Torres, 1997, 1998 a et b). 
Pourtant, comme nous l'avons vu dans cette enquête, pour que le travail en dyades soit fructueux, il ne suffit pas de garantir que les élèves soient assis deux par deux. Il faut choisir les bons critères pour la constitution des paires; concevoir des tâches qui facilitent les interactions horizontales et la réflexion en commun; donner des consignes de travail qui favorisent l'acceptation d'un contrat didactique innovateur et permettent aux maîtres de s'intéresser aux productions et aux processus de raisonnement des élèves; et qui permettent à ces derniers de ressentir par là que leur pensée est prise au sérieux sans que les erreurs normales de tout parcours d'apprentissage ne soient dramatisées.

Le travail en dyades s'est montré suffisamment fructueux pour susciter, nouvellement, des collaborations entre chercheurs et enseignants. Un pont s'est construit entre la recherche fondamentale et la recherche appliquée. Une voie s'ouvre maintenant pour poursuivre l'observation des processus d'apprentissage en tenant compte des composantes, complexes, de leur contexte «naturel» à l'école.

\section{Remerciements}

Nous remercions sincèrement Luc-Olivier Pochon pour son soutien de tous ordres au long de cette recherche. Notre gratitude va aux enseignants et aux élèves qui ont été nos partenaires et ont collaboré à nos observations et à notre réflexion.

\section{Références bibliographiques}

Abrantes, P., Serrazina, L. \& Oliveira, I. (1999). A matemática na educação básica. Lisboa: Ministério da Educação, Departamento de Educação Básica.

Allal, L. \& Ducrey, G. P. (2000). Assessment of - or in - the zone of proximal development. Learning and Instruction, 10(2), 137-152.

Ames, G. \& Murray, F. (1982). When two wrong make a right: promoting cognitive change by social conflict. Developmental Psychology, 18(6), 894-897.

Balacheff, N. \& Laborde, C. (1985). Langage symbolique et preuves dans l'enseignement mathématique: une approche sociocognitive. In G. Mugny (Ed.), Psychologie social du développement cognitif (pp.203-223). Berne: Peter Lang.

Balacheff, N. (1982). Preuve et démonstration en mathématiques au collège. Recherches en Didactiques des Mathématiques, 3(3), 261-304.

Benavente, A. (1990). Escola, Professoras e Processos de Mudança. Lisboa: Livros Horizonte.

Benavente, A. (1992). Ganhar tempo, perder tempo. Diário de Notícias, 21 de Novembro de 1992.

Blaye, A. (1987). Organisation du produit de deux ensembles: influence des interactions sociales entre pairs sur les procédures de résolution et les performances individuelles. European Journal of Psychology of Education, 1(4), 29-44.

Blaye, A. (1988). Mécanismes générateurs de progrès lors de la résolution à deux d'un produit de deux ensembles par des enfants de 5-6 ans. In A.-N. Perret-Clermont \& M. Nicolet (Eds.), Interagir et connaître - enjeux et régulations sociales dans le développement cognitif (pp.41-54). Fribourg: Del Val. 
Botelho, D. (1991). Interacção e Desenvolvimento: Análise Procedimental de Situações de Resolução e Co-Resolução de um Problema em Cinco Grupos de Idades. Análise Psicológica, IX(3/4), 435-443.

Brousseau, G. (1988). Le contract didactique: Le milieu. Recherches en Didactiques des Mathématiques, 9(3), 309-336.

Brun, J. (1975). Education mathématique et développement intellectuel recherche à propos de l'enseignement rénové de la mathématique sur des enfants en fin de scolarité primaire. Thèse de doctorat non publiée en psychologie et sciences sociales, Univesité de Lyon II.

Carraher, T., Carraher, D. \& Schliemann, A. (1989). Na vida dez, na escola zero. S. Paulo, Brésil: Cortex Editora.

Carugati, F. \& Mugny, G. (1985). La théorie du conflit sociocognitif. In G. Mugny (Ed.), Psychologie de développement cognitif. (pp. 57-70). Berne: Peter Lang.

César, M. (1994a). O papel da interacção entre pares na resolução de tarefas matemáticas: trabalho em diade vs. trabalho individual em contexto escolar. Thèse de doctorat, Université de Lisbonne, texte polycopié.

César, M. (1994b), Factores psico-sociais e equações, Actas do ProfMat 94, (pp.82-92). Lisboa: Associação de Professores de Matemática.

César, M. (1995), Interacção entre pares e resolução de tarefas matemáticas, Actas do VI Encontro de Investigação em Educação Matemáica (pp.225-240). Lisboa: Associação de Professores de Matemática.

César, M. (1997). Investigação, Interacções entre Pares e Matemática. Actas do VIII Seminário de Investigação em Educação Matemática. (pp. 7-33). Lisboa: APM.

César, M. (1998). Y se aprendo contigo? Interacciones entre parejas en el aula de matemáticas. Uno, 16, 11-23.

César, M. (2000a). Interacções sociais e apreensão de conhecimentos matemáticos: A investigação contextualizada. In J. P. Ponte \& L. Serrazina (Eds.), Educação Matemática em Portugal, Espanha e Itália: Actas da Escola de Verão - 1999 (pp. 5-46). Lisboa: SEM/ SPCE.

César, M. (2000b). Interaction and Knowledge: Where are we going in the 21 st century?. In M. A. Clements, H. H. Tairab \& W. K. Yoong (Eds.), Science, Mathematics and Technical Education in the 20th and 21st Centuries (pp. 317-328). Bandar Seri Begawan: Universiti Brunei Darussalam.

César, M. (2000c). Interacções na aula de Matemática: Um percurso de 20 anos de investigação e reflexão. In C. Monteiro et al. (Eds.), Interacções na Aula de Matemática (pp. 13-34). Viseu: SPCE - Secção de Educação Matemática.

César, M., Camacho, H. \& Marcelino, T. (1991). Desenvolvimento cognitivo e sucesso escolar num meio socio-culturalmente desfavorecido. Aprendizagem e Desenvolvimento, III (13/14), 149-152.

César, M. \& Esgalhado, A. (1986). Symbolisme et développement cognitive - quelques donnés à propos de la traduction et de ladaptation de l'E.C.D.L. Poster présenté au 8e Cours Avancé de la Fondation des Archives Jean Piaget, Genève 22-26 septembre 1986.

César, M. \& Esgalhado, A. (1987). L'E.C.D.L. et les différences individuelles. Poster presenté au 8e Cours Avancé de la Fondation des Archives Jean Piaget, Genève 21-25 septembre 1987.

César, M. \& Esgalhado, A. (1988). Desenvolvimento cognitivo e sucesso escolar. Actas do Encontro Internacional de Intervenção Psicológica na Educação (pp.365-371). Porto: APPORT.

César, M. \& Esgalhado, A. (1991). Desenvolvimento cognitivo e percurso escolar. Revista de Educação, $I I(1)$, 57-61.

César, M. \& Torres, M. (1998a). Pupils' Interactions in Maths Class. CIEAEM 49 Proceedings, pp. 76-85. Setúbal: ESE de Setúbal.

César, M. \& Torres, M. (1998b). Actividades em Interacção na Sala de Aula de Matemática. Actas do VI Encontro de Investigação em Educação Matemática, Portalegre: (pp. 7187).SPCE. 
Chevallard, Y., Guidoni, P, Nelson, L. \& Schubauer-Leoni, M.-L. (1988). Mediations et rémédiations didactiques. Interactions Didactiques, no 9 . Genève, Neuchâtel: Universités de Genève et Neuchâtel.

Doise, W., Mugny, G. \& Perret-Clermont, A.-N. (1975). Social interaction and the development of cognitive operations. European Journal of Social Psychology, 5(3), 367-383.

Doise, W., Mugny, G. \& Perret-Clermont, A.-N. (1976). Social interaction and cognitive development: further evidence. European Journal of Social Psychology, 6(2), 245-247.

Donaldson, M. (1978). Children's minds. New-York: W.W. Norton.

Flieller, A. (1986). La coéducation de l'intelligence. Nancy: Presses Universitaires de Nancy.

Flieller, A. (1990). À côté des conflits socio-cognitifs. Psychologie Scolaire, 71, 20-32.

Forman, E. (1989) The role of peer interaction in the social construction of mathematical knowledge. International Journal of Educational Research, 13, 55-69.

Forman, E. (1995) Learning in the context of peer collaboration: a pluralistic perspective on goals and expertise. Cognition and Instruction, 13(4), 549-564.

Gilly, M., Fraisse, J. \& Roux, J.-P. (1988). Résolution de problèmes en dyade et progrès cognitifs chez des enfants de 11 à 13 ans: dynamiques interactives et socio-cognitives. In A.$\mathrm{N}$. Perret-Clermont \& M. Nicolet (Eds), Interagir et connaître - enjeux et régulations sociales dans le développement cognitif(pp.73-92). Fribourg: Del Val.

Gilly, M. \& Roux, J.-P. (1984). Efficacité comparée du travail individuel et du travail en interaction socio-cognitive dans l'appropriation et la mise en oeuvre de règles de résolution chez des Enfants de 11-12 ans. Cahiers de Psychologie Cognitive, 4(2), 171-188.

Giosue, F. (1991). Quanti anni hanno la maestra e il capitano? Appoggio psicosociale alla costruzione della risposta a problemi assurdi in contesto scolastico. Thèse en sciences de l'éducation, Università di Bologna, texte polycopié.

Grossen, M. (1988). Lintersubjectivité en situation de test. Fribourg: Del Val.

Grossen, M. \& Perret-Clermont, A.-N. (1984). Some elements of a social psychology of operational development of the child. The Quarterly Newsletter of the Laboratory for Comparative Human Cognition, 6(3), 51-57.

Grossen, M. \& Py, B. (Eds.). (1997). Pratiques sociales et médiations symboliques. Berne: Peter Lang.

Healy, L., Hoyles, C. \& Sutherland, R. (1990). The Role of Peer Group Discussion in Mathematical Environments. Research report. London: Institute of Education University of London, Department of Mathematics, Statistics and Computing.

Howe, C., Tolmie A., Greer K. \& Mackenzie, M. (1995) Peer collaboration and conceptual growth in physics: task influences on children's understanding of heating and cooling. Cognition and Instruction, 13 (4), 423-503.

Iannaccone, A. (1990). Rapporto di ricerca: Attualizzazione del giudizi operatori e delle capacità logico matematiche in bambini di età prescolarelscolare in interazione con adulti che hanno o assumono ruolli diversi. Salermo: Universités de Salerne et de Neuchâtel,Séminaire de Psychologie.

Johnson, D. W. \& Johnson, R. T. (1989). Cooperation and competition: A meta-analysis of the research. Hillsdale, NY: Erlbaum.

Jonckheere, A. R. (1954). A distribution-free k-sample test against order alternatives. Biometrica, 41, 133-145.

Kumpulainen, K. \& Mutanen, M. (1999). The situated dynamics of peer group interaction: an introduction to an anlytic framework. Learning and Instruction, 9(5), 449-473.

Leach, C. (1979). Introduction to statistics: a nonparametric approach for the social sciences. Chichester, New York [etc.]: J. Wiley and Sons.

Light, P. (1986). Context, conservation and conversation. In P. L. M. Richard (Ed.), Children of social worlds. Development in a social context. Cambridge: Polity Press.

Light, P., Gorsuch, C. \& Newmann, J. (1987). Why do you ask? Context and communication in the conservation task. European Journal of Psychology of education, 2, 73-82. 
Martins, M.A. \& Neto, F.C. (1990). A Influência dos Factores Sociais Contextuais na Resolução de Problemas. Análise Psicológica, VIII(3), 265-274.

Moll, L.C. (1990). Vygotsky and Education. Cambridge UK: Cambridge University Press.

Mugny, G. (Ed.). Psychologie sociale du développement cognitif. Berne: Peter Lang, Collection Exploration.

Nicolet, M. (1995). Dynamiques relationnelles et développement cognitif. Lausanne; Paris: Delachaux et Niestlé.

Peixoto, F. \& Monteiro, V. (1999). Interacçōes sociais, desenvolvimento e aprendizagem: O papel do estatuto do par e da mediação semiótica. Análise Psicológica, XVII(1), 9-17.

Perret-Clermont, A.-N. (1979/ version augmentée 1996). La construction de l'intelligence dans l'interaction sociale. Berne: Peter Lang, Collection Exploration.

Perret-Clermont, A.-N. (1980). Recherche en psychologie sociale expérimentale et activité éducative: deux élaborations symboliques, deux pratiques, qui peuvent être complémentaires. Revue Française de Pédagogie, 93, 30-38.

Perret-Clermont, A.-N. (1982). Approaches in the Social Psychology of Learning and Group Work. In P. Stringer (Ed.), Confronting Social Issues (pp.97-122). London: Academic Press.

Perret-Clermont, A.-N. \& Nicolet, M. (1988). Interagir et Connaître - Enjeux et régulations sociales dans le développement cognitif. Fribourg: Del Val.

Perret-Clermont, A. N., Perret, J. F., \& Bell, N. (1991). The social construction of meaning and cognitive activity in elementary school children. In L. B. Resnick, J. M. Levine, \& S. D. Teasley (Eds.), Perspectives on socially shared cognition (pp. 41-62). Washington, D.C.: American Psychological Association.

Piaget, J. (1977/95). Sociological Studies. London: Routledge. [original en français en 1965, élargi en 1977]

Pochon, L.O. (1991) Statistiques et sciences humaines. Notes de travail. Dossier de Psychologie no 38, Neuchâtel: Université de Neuchâtel.

Pochon, L.O. (1997) ANASTAT: Un système dédié à la gestion et à l'analyse de données paramétriques. Dossier de Psychologie no 48, Neuchâtel: Université de Neuchâtel et http://www.unine.ch/psy/stat/anastat.zip.

Pléty, R. (1996) L'apprentissage coopérant. Lyon: Presses Universitaires de Lyon.

Rogoff, B. \& Wertsch, J. V. (1984). Children's Learning in the Zone of Proximal Development. San Francisco: Jossey-Bass.

Rommetveit, R. (1976). On the architecture of intersubjectivity. In L. H. Strickland, K. J. Gergen, \& F. J. Aboud (Eds.), Social psychology in transition. New York: Plenum Press.

Säljö, R., \& Wyndhamn, J. (1987). The formal setting as context for cognitive activities. An empirical study of arithmetic operations under conflicting premisses for communication. European Journal of Psychology of Education, II(3), 233-245.

Säljö, R. (1991). Piagetian controversies, cognitive competence, and assumptions about human communication. Educational Psychology Review, 3(2), 117-126.

Saxe, J.G. (1989). Selling Candy: a study of cognition in context. The Quarterly Newsletter of the Institute for Comparative Human Development, 11(1-2), 19-22.

Schubauer-Leoni, M. L. (1986a). Le contrat didactique: un cadre interprétatif pour comprendre les savoirs manifestés par les élèves en mathématiques. European Journal of Psychology of Education, 1(2), 139-153.

Schubauer-Leoni, M.L. (1986b). Le contract didactique dans l'élaboration d'écritures symboliques par des élèves de 8-9ans. Interactions Didactiques, no 7. Genève, Neuchâtel: Universités de Genève et Neuchâtel.

Schubauer-Leoni, M. L. (1988). Des recherches en didactique des mathématiques avec un regard de psychologue social des situations d'enseignement. Communication présentée au Colloque Psychologie des Constructions Cognitives et Education, Aix-en-Provence, Décembre 1988. 
Schubauer-Leoni, M. L. (1990). Ecritures additives en classe et en dehors de la classe: une affaire de contexte. Résonances, 6, 16-18.

Schubauer-Leoni, M. L. \& Perret-Clermont, A.-N. (1980). Interactions sociales et représentations symboliques dans le cadre de problèmes additifs. Recherches en Didactiques des $\mathrm{Ma}$ thématiques, 1(3), 297-350.

Schubauer-Leoni, M. L. \& Perret-Clermont, A.-N. (1985). Interactions sociales dans l'apprentissage de connaissances mathématiques chez l'enfant. In G. Mugny (Ed.), Psychologie sociale du développement cognitif. (pp.225-250). Berne: Peter Lang,.

Schubauer-Leoni, M.L. \& Perret-Clermont, A.-N. (1988). Représentations et significations des savoirs scolaires. European Journal of Psychology of Education (Special Issue), 55-62.

Schubauer-Leoni, M.L. \& Perret-Clermont, A.-N. (1997). Social Interactions and Mathematics Learning. In T. Nunes \& P. Bryant (Eds.) Learning and Teaching Mathematics: An International Perspective (pp.265-283). Hove: Psychology Press.

Slavin, R. E. (1980). Cooperative learning. Review of Educational Research, 50(2), 315-342.

Slavin, R. E. (1990). Research on Cooperative Learning: Consensus and Controversy. Educational Leadership, 47(4), 52-54.

Smith, L. (1996). The social construction of rational understanding. In A. Tryphon \& J. Vonèche (Eds.), Piaget - Vygotsky. The Social Genesis of Thought (pp. 107-123). Hove: Psychology Press.

Tudge, J. (1985). The effect of social interaction on cognitive developpement: how creative is conflict? The Quarterly Newsletter of the Laboratory of Comparative Human Cognition, 7(2), 33-34.

Tudge, J. (1989). When collaborative leads to regression: Some negative consequences of socio-cognitive conflict. European Journal of social Psychology, 19, 123-138.

Van der Linden, J., Erkens, G., Schmidt, H. \& Renhard, P. (2000). Collaborative learning. In P.R.J. Simons, J.L. van der Linden \& T. Duffy (Eds.), New Learning. Dordrecht: Kluwer Academic Publisher.

Vergnaud, G. (1986). Psicologia do desenvolvimento cognitivo e didáctica das matemáticas. Um exemplo: as estruturas aditivas. Análise Psicológica, V(1), 75-90.

Vygotsky, L.S. (1962). Thought and Language. Cambridge: MIT Press [Original publié en $1934]$

Vygotsky, L.S. (1978). Mind and Society: The development of higher psychological processes. Cambridge, MA: Harvard University Press. [Original publié en 1932]

Vygotsky, L.S. (1985). Le problème de l'enseignement et du développement à l'âge scolaire. In B. Schneuwly \& J.-P. Bronkart (Eds.), Vygotsky aujourd'hui (pp.95-117). Neuchâtel: Delachaux et Niestlé.

Wertsch, J.V. (1991). Voices of Mind. A sociocultural approach to mediated action. Hempstead: Havester/Wheatsheaf. 


\section{Arbeitsmethoden und -verhalten beim Lösen von Algebraaufgaben in Dyaden bei portugiesischen Schülerinnen und Schülern}

\section{Zusammenfassung}

Die vorliegende Studie stammt aus Portugal, wo es den Mathematiklehrern der Sekundarschule ernsthafte Probleme bereitet, ihre Schülerinnen und Schüler zu motivieren. Viele sind an Mathematik uninteressiert und fallen bei Prüfungen durch. Spezifische pädagogische Verfahren, die in experimentellen Fallstudien angewendet wurden, ermöglichten es uns, die verschiedenen Arbeitsmethoden der Schüler zu beobachten, wenn sie zu zweit oder alleine ein Algebraproblem lösten. Die Studie zeigt, welche Rolle die Art des Verhältnisses der Schüler zueinander in jedem Paar spielt und wie sich dieses Verhältnis zur Leistung im Verstehen und Lösen der Aufgabe verhält. Die Begeisterung unter den Schülern wuchs während dieser Studie. Sobald die Schüler im Dialog miteinander arbeiteten und sozio-kognitive Konflikte entstehen konnten, verbesserten die Schülerinnen und Schüler ihre Haltung gegenüber der Mathematik.

\section{Modalità e procedure di lavoro diadico di allievi portoghesi su problemi di algebra}

\section{Riassunto}

La ricerca presentata qui di seguito si svolge nel contesto della scuola obbligatoria portoghese. Essa si interessa particolarmente alle difficoltà relative all'insegnamento della matematica che fanno sì che sistematicamente una proporzione importante di allievi risulti poco motivata per tale disciplina e consegua risultati negativi. Una serie di attività pedagogiche, concepite sotto forma di studio di casi ispirati a una metodologia di tipo quasi-sperimentale che rende possibile dei confronti, ha permesso di osservare le differenti reazioni degli allievi intenti a risolvere, soli o in coppia, dei problemi d'algebra. I risultati, da un lato consentono una migliore comprensione del ruolo delle relazioni diadiche ai fini del successo nella comprensione e nella gestione del compito; e dall'altro mettono in evidenza il gusto per l'attività che si sviluppa negli allievi coinvolti in una spirale di dialoghi e di conflitti socio-cognitivi, e che finisce di conseguenza con il modificare i loro atteggiamenti nei confronti della matematica. 


\section{Methods and behavior of Portuguese students solving algebra problems in pairs}

\section{Summary}

The study below took place in Portugal, where secondary school math teachers encountered serious problems when attempting to motivate their students, a large number of whom were indifferent to, and failed, math. Specific pedagogical procedures, designed as experimental case studies facilitating comparisons, allowed us to observe the various approaches the children took to algebra problems as they worked alone or in pairs. The study made manifest the role played by the modalities of relations between the students in each dyad relative to the group's success in understanding and completing its task. A growing enthusiasm among the students also emerged during the study; once they initiated a dialogue, a series of socio-cognitive interactions, or even conflicts, their attitude to mathematics improved. 